\title{
Glomerular Diseases in Children - A Review of 27 Cases Recorded at a Single Centre in Eastern Nepal
}

\author{
Shrestha P ${ }^{1}$, Bhatta NK ${ }^{2}$,Tiwari A ${ }^{3}$, Singh R ${ }^{4}$, Kalakhetti BK ${ }^{5}$, Majhi $\mathbf{S}^{6}$, Paudel P ${ }^{7}$, Sinha $\mathrm{A}^{4}$ \\ ${ }^{1}$ Dr. Pramod Shrestha MD (Assistant Professor), Department of Pediatrics and Adolescent Medicine. ${ }^{2}$ Dr Nisha \\ Keshari Bhatta MD (Associate Professor),Department of Pediatrics and Adolescent Medicine. ${ }^{3}$ Dr Awadesh Tiwari \\ MD (Associate Professor), Department of Radiodiagnosis and Imaging. ${ }^{4} \mathrm{Dr}$ Rupa Singh MD (Professor), Department \\ of Pediatrics and Adolescent Medicine. ${ }^{5}$ Dr BalKrishna Kalakheti MD (Assistant Professor), Department of Pediatrics \\ and Adolescent Medicine. ${ }^{6}$ Dr Shankar Majhi MD (Additional Professor), Department of Biochemistry. ${ }^{7}$ Dr Prakash \\ Paudyal (Senior Resident), Department of Pediatrics and Adolescent Medicine. ${ }^{8}$ Dr Arvind Sinha MD (Additional \\ Professor), Department of Pathology. All from BP Koirala Institute of Health Sciences, Dharan, Nepal.
}

Address of Correspondence: Pramod Shrestha, MD. E-mail: prmd_shrestha@yahoo.com

\begin{abstract}
Glomerular disease remains an important cause of renal disease in children. We retrospectively assessed the clinical features and histopathological diagnoses in kidney biopsy specimens in children $(n=27)$. The medical records and histopathological diagnoses between 2003 and 2006 were reviewed. In each patient, clinical presentation, age at onset of disease, gender, presence of nephrotic range proteinuria, hematuria, hypertension and histopathological diagnoses were evaluated. SLE nephritis $(n=8)$ and membranoproliferative glomerulonephritis (MPGN) $(n=8)$ were the most frequent glomerular diseases and nephrotic syndrome $(\mathrm{n}=21)$ was the frequent clinical manifestation.
\end{abstract}

Key words: Renal biopsy, Glomerular diseases

\section{Introduction}

"Glomerular disease" indicates that the initial and major point of impact within the renal tissue is in the glomerulus. ${ }^{1}$ The clinical presentation may vary from one patient to another. On the other hand the same clinical presentation may be associated with many glomerular diseases. In this study, we evaluated the various types of glomerular diseases, their clinical presentations and histopathological diagnoses based on kidney biopsies received in 4 years duration.

\section{Patients and methods}

The medical records and histopathological diagnoses of kidney biopsy specimens between 2003 and 2006 were reviewed. Biopsies were evaluated by light microscope. Each biopsy specimen was stained with periodic acid-schiff (PAS) reagent and periodic acid silver methenamine (PASM). Indications for renal biopsy were:-

1. Onset of Nephrotic Syndrome at $<1$ year or $>8$ years of age.

2. Steroid resistant Nephrotic Syndrome or steroid dependent Nephrotic Syndrome.
3. Frequent relapse Nephrotic Syndrome.

4. Renal involvement in systemic diseases.

5. Persistent proteinuria and/ or hematuria.

6. Persistent azotemia.

7. Rapid deterioration in renal functions.

Nephrotic proteinuria was defined as protein excretion rate $\geq 40 \mathrm{mg} / \mathrm{m}^{2} / \mathrm{hr}$, hematuria as more than 5 RBCs per high power field in light microscopy, and hypertension as systolic and diastolic blood pressure, equal to or more than $95^{\text {th }}$ percentile for age and gender on three consecutive days. Nephrotic syndrome was defined as nephrotic range proteinuria, edema, hypoalbuminemia $(<2.5 \mathrm{~g} / \mathrm{dl})$, and hyperlipidemia. ${ }^{1}$ The pathological diagnoses were based on the WHO classification of glomerular diseases. ${ }^{2}$ Definitions of clinical presentations were as follows:-

1. Asymptomatic proteinuria or hematuria.

2. Non-nephrotic proteinuria or persistent hematuria or both in absence of other evidence of renal disease. 
3. Acute glomerulonephritis (AGN).

4. Sudden onset of varying degrees of hypertension, edema, gross or microscopic hematuria, oliguria and azotemia.

5. Rapid Proliferative Glomerulonephritis (RPG).

6. A variant of AGN with more severity and potential for progression to end stage renal disease within weeks to months.

7. Chronic glomerulonephritis (CGN).

8. Protracted course of varying degrees of hypertension, edema, proteinuria, hematuria, and azotemia, with irreversible progression to end stage renal disease.

9. Nephrotic syndrome.

10. Heavy proteinuria, hypoalbunemia, edema, and hyperlipidemia.
SLE nephritis $(\mathrm{n}=8)$ were the commonest glomerular diseases. Correlations between glomerular diseases and their clinical presentations are highlighted in Table 2. Nephrotic syndrome $(n=21)$ was the common clinical presentation of glomerular disease.

\section{Discussion}

In our study, SLE nephritis and membranoproliferative glomerulonephritis (MPGN) were the most frequent glomerular diseases in children. Nephrotic syndrome was the frequent clinical manifestation of glomerular diseases in children. Data shows nephrotic syndrome was the most common clinical manifestation (77\%) of glomerular disease in children. ${ }^{3}$ Various studies have shown minimal change disease (MCD) as the most common form of nephrotic syndrome in children., ${ }^{4,5,7}$ However, in our study, we found MPGN as the commonest cause of nephrotic

Table 1: Types of Glomerular Diseases and their Major Clinical Presentations.

\begin{tabular}{|l|c|c|c|c|c|c|}
\hline $\begin{array}{l}\text { Glomerular } \\
\text { Disease }\end{array}$ & $\begin{array}{c}\text { Number } \\
(\mathbf{\%})\end{array}$ & $\begin{array}{c}\text { Mean } \\
\text { Age (yr) }\end{array}$ & $\begin{array}{c}\text { Female } \\
\mathbf{( \% )}\end{array}$ & $\begin{array}{c}\text { Nephrotic } \\
\text { Proteinuria } \\
(\mathbf{\%})\end{array}$ & $\begin{array}{c}\text { Hematuria } \\
\mathbf{( \% )}\end{array}$ & Hypertension (\%) \\
\hline Total & $27(100)$ & 9.7 & 66.6 & 88.8 & 80 & 66.6 \\
\hline MPGN $^{\text {a }}$ & $8(29.6)$ & 9.2 & 50 & 100 & 62.5 & 62.5 \\
\hline SLE & $8(29.6)$ & 10.3 & 87.5 & 75 & 37.5 & 75 \\
\hline MCD & $4(14.8)$ & 9.5 & 50 & 100 & 25 & 50 \\
\hline FSGS & $4(14.8)$ & 10.5 & 75 & 100 & 100 & 75 \\
\hline HSP & $2(7.4)$ & 6.5 & 100 & 100 & 100 & 50 \\
\hline ECGN & $1(3.7)$ & 14.0 & 0 & 0 & 100 & 100 \\
\hline
\end{tabular}

$M P G N$ membranoproliferative glomerulonephritis; SLE systemic lupus erythromatosus; $M C D$ minimal change disease; FSGS focal segmental glomerulosclerosis; HSP Henoch-Schoenlein purpura; ECGN endocapillary glomerulonephritis.

${ }^{\text {a }}$ Further classification not done.

Table 2: Correlation between Glomerular Diseases and their Clinical Presentations.

\begin{tabular}{|l|c|c|c|c|}
\hline Clinical Manifestation $(\rightarrow)$ & NS & AGN & Hematuria & Hypertension \\
\hline Glomerular Disease $(\downarrow)$ & & & & \\
\hline MPGN & 8 & 0 & 5 & 5 \\
\hline SLE & 5 & 3 & 3 & 6 \\
\hline MCD & 4 & 0 & 1 & 2 \\
\hline FSGS & 4 & 0 & 4 & 3 \\
\hline HSP & 0 & 2 & 2 & 2 \\
\hline ECGN & 0 & 1 & 1 & 1 \\
\hline
\end{tabular}

$M P G N$ membranoproliferative glomerulonephritis; SLE systemic lupus erythromatosus; $M C D$ minimal change disease; FSGS focal segmental glomerulosclerosis; HSP Henoch-Schoenlein purpura; ECGN endocapillary glomerulonephritis.

\section{Results}

Twenty seven kidney biopsy specimens received during study period $(2003-2006)$ were reviewed. Table 1 depicts the type of glomerular diseases diagnosed and their major clinical manifestations. MPGN $(n=8)$ and syndrome. This could be explained by the fact that those children with clinical and laboratory evidences of MCD were not subjected to kidney biopsy. On the other hand the higher incidence of MPGN could be due to low socioeconomic conditions in this area. Simon et 
$\mathrm{al}^{8}$ reported a decline in rheumatic fever was associated with a parallel decline in MPGN and post-streptococcal GN and such decline was attributed to an improved standard of living, better public health, and the early antibiotic treatment of pharyngeal infections. Our study showed that nephrotic syndrome remains the most common clinical presentation of various glomerular diseases. Table 3 depicted the comparison of our results with other studies.

In this study, among patients with clinical presentation of AGN, SLE nephritis was the most frequent cause, followed by HSP nephritis, and ECGN. the frequent cause of nephrotic syndrome in children. SLE nephritis was the frequent cause of AGN and needs to be considered as differential in children with PSGN.

\section{References}

1. Bernstein J, Edelmann CM. Glomerular diseases: Introduction and classification. In Edelmann CM, editor. Pediatric kidney disease, (2 ${ }^{\text {nd }}$ ed.) Little Brown Boston, 1992: 1181-88.

2. Churg J, Sobin LH. Renal disease: classification and atlas of glomerular diseases. Igaku-Shoin Tokio, 1982: 3-19.

Table 3: Glomerular diseases in various studies

\begin{tabular}{|c|c|c|c|c|c|c|c|c|}
\hline & \multicolumn{2}{|c|}{ Our patients } & \multicolumn{2}{|c|}{ Bhimma et al } & \multicolumn{2}{|c|}{ Srivastava $e a l^{\mathrm{b} 6}$} & \multicolumn{2}{|c|}{ Madani et $\mathrm{ll}^{\mathrm{c} 7}$} \\
\hline & n & $\%$ & $\mathbf{n}$ & $\%$ & $\mathbf{n}$ & $\%$ & n & $\%$ \\
\hline Total & 27 & 100 & 545 & 100 & 148 & 100 & 330 & 100 \\
\hline MCD & 4 & 14.8 & $233^{a}$ & 42.7 & - & 52.7 & 90 & 27.2 \\
\hline FSGS & 4 & 14.8 & 136 & 25 & - & 23 & 84 & 25.5 \\
\hline FGGS & - & - & - & - & - & 0.7 & 29 & 8.4 \\
\hline DMP & - & - & 18 & 3.3 & - & 12.2 & 24 & 7.2 \\
\hline MPGN & 8 & 29.6 & 21 & 3.8 & - & 9.5 & 23 & 7 \\
\hline MGN & - & - & 26 & 4.8 & - & 1.9 & 19 & 5.7 \\
\hline SLE & 8 & 29.6 & - & - & - & - & 15 & 4.6 \\
\hline FGM & - & - & - & - & - & - & 12 & 3.6 \\
\hline ECGN & 1 & 3.7 & - & - & - & - & 3 & 1 \\
\hline IgAN & - & - & - & - & - & - & 2 & 0.6 \\
\hline CRES & - & - & - & - & - & - & 2 & 0.6 \\
\hline HSP & 2 & 7.4 & - & - & - & - & 1 & 0.3 \\
\hline Others & - & - & 111 & 20.4 & - & - & 26 & 7.9 \\
\hline
\end{tabular}

${ }^{a}$ Including 65 cases with presumed MCD. ${ }^{\mathrm{b}}$ Primary Nephrotic syndrome only. ${ }^{\mathrm{c}}$ Clinical presentation of Nephrotic syndrome only. $M C D$ minimal change disease; FSGS focal segmental glomerulosclerosis; FGGS focal global glomerulosclerosis; $D M P$ diffuse mesangial proliferation; $M P G N$ membranoproliferative glomerulonephritis; $M G N$ membranous glomerulonephritis; $S L E$ systemic lupus erythromatosus; $F G N$ focal glomerulonephritis; $E C G N$ endocapillary glomerulonephritis; $\operatorname{Ig} A N$ IgA nephropathy; CRES crescentric glomerulonephritis; HSP Henoch-Schoenlein purpura.

In one study, SLE nephritis was the common cause of AGN, however their patients were adults. ${ }^{9}$ MPGN was the third cause of AGN in glomerular disease ${ }^{7}$ which is in contrary to our study where all patients with MPGN had presentation of nephrotic syndrome. The low frequency of ECGN in this study may be due to the fact that children with typical presentation of poststreptococcal glomerulonephritis (PSGN) had not been biopsied at all. HSP is the clinical diagnosis and renal biopsy is reserved only for those cases with unfavorable clinical course with renal involvement or persistent renal affection.

In conclusion, our observations showed nephrotic syndrome as the common clinical presentation of various glomerular diseases, and we found MPGN as
3. Al-Rasheed SA, Al-Mugeriren MM, Al-Salloum AA, Al-Sohaibani MO. Childhood renal disease in Saudi-Arabia. A clinico-pathological Study of 167 cases, Int Urol Nephrol 1996; 28(5): 607-13.

4. ISKDC. The primary nephrotic syndrome in children. Identification of patients with minimal change nephrotic syndrome from initial response to prednisone. J Pediatr 1981; 98: 561-64.

5. Bhimma R, Coovadia HM, Adhikari M. Nephrotic syndrome in South African children: changing perspectives over 20 years. Pediatr Nephrol 1997; 11: 429-34.

6. Srivastava T, Simon SD, Alon US. High incidence of FSGS in nephrotic syndrome in children. Lancet 1999; 1:1299-302. 
7. Madani A, Fahimi D, Esfehani ST et al. Glomerular disease in Iranian children: clinico-pathological correlations. Pediatr nephrol 2003; 18: 925-28.

8. Simon P, Ramee MP, Boulahrouz R. Epidemiologic data of primary glomerular diseases in western France. Kidney Int 2004; 66: 905-8.
9. Jennette JC. Prologue to Section II. In: Jennette JC, Olson JL, Schwartz MM, Silva FG (eds), Heptinstall's pathology of the kidney, $\left(5^{\text {th }}\right.$ ed $)$ Little Brown, Boston, 1998: 182.

\section{ERRATUM}

The following corrections are to be made in the last issue of this journal.

In the original article entitled "Distribution of Serotypes and Antimicrobial Resistance of Streptococcus Pneumoniae in a Children's Hospital in Nepal" by Sherchand JB et al., published in Volume 28 Issue 2, JulyDecember 2008 of Journal of Nepal Paediatrics Society page number 45.

Instead of being (1) "Ashish Raj Joshi; (MSc Medical Microbiology), College of Science and Technology, Kalimati" should be read as: - "Ashish Raj Joshi (MSc Medical Microbiology), Kathmandu College of Science and Technology, Kalimati”

And instead of being (2) "Jyoti Amatya; (MSc Medical Microbiology), Assistant Professor in Microbiology: College of Science and Technology, Kalimati, Nepal." should be read as: - "Jyoti Amatya; (MSc Medical Microbiology), Assistant Professor in Microbiology: Kathmandu College of Science and Technology, Kalimati, Nepal."

The editorial board regrets this error and assures you that it shall not happen in future.

\section{- Editorial Board}

